\title{
Research Paper \\ The Effect of Purslane Ointment on Chronic Musculoskeletal Pain in the Elderly
}

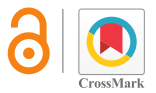

\author{
Amirreza Nasirzadeh ${ }^{1} \oplus$, Mahdi Basiri Moghadam², ${ }^{*}$ Kokab Basiri Moghadam $^{3}{ }^{(0)}$
}

1. Student Research Committee, Gonabad University of Medical Sciences, Gonabad, Iran

2. Nursing Care Research Center, Iran University of Medical Sciences, Tehran, Iran.

3. Department of Internal and Surgical Nursing and Pediatrics, School of Nursing and Midwifery, Gonabad University of Medical Sciences, Gonabad, Iran.

\begin{tabular}{|c|c|}
\hline $\begin{array}{l}\text { Use your device to scan } \\
\text { and read the article online }\end{array}$ & Citation Nasirzadeh A, Basiri Moghadam M, Basiri Moghadam K. [The Effect of Purslane Ointment on Chronic Musculoskele- \\
\hline aristing & $\begin{array}{l}\text { tal Pain in the Elderly (Persian)]. Quarterly of "The Horizon of Medical Sciences". 2019; 25(3):244-255. https://doi.org/10.32598/ } \\
\text { hms.25.3.244 }\end{array}$ \\
\hline 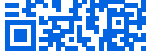 & dol'https://doi.org/10.32598/hms.25.3.244 \\
\hline
\end{tabular}

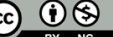

Received: 03 Feb 2019

Accepted: 20 May 2019

Available Online: $01 \mathrm{Jul} 2019$

Key words:

Purslane, Elderly,

Musculoskeletal pain,

Geriatric nursing,

Pain relief

\section{A B STRACT}

Aims Chronic musculoskeletal pain is among the major problems of old age. An effective nursing intervention in relieving pain is the use of local herbal ointments. The present study evaluated the effect of purslane ointment on chronic musculoskeletal pain in the elderly.

Methods \& Materials This was a randomized, blinded clinical trial study and conducted in 2018, on 60 elderly residents of Gonabad City, Iran. The patients were recruited by convenience sampling method. The samples were randomly assigned to the purslane and placebo groups. The instrument used in this study was McGill pain questionnaire; its validity and reliability were confirmed in previous studies. The intervention was conducted for two weeks. The obtained data were analyzed by SPSS and at a significance level of $\mathrm{P}<0.05$.

Findings The collected results suggested that the purslane ointment could effectively reduce the sensory and emotional dimensions of pain, compared with placebo. The severity of pain in the elderly was significantly lower in the purslane group after two weeks of intervention, compared to the Pre-test phase. The mean score of pain in two sensory and emotional dimensions was not significantly different between the two genders.

Conclusion The use of purslane ointment is effective in reducing musculoskeletal pain in the elderly; therefore, the use of this plant can be considered as a safe and cost-effective intervention by nurses.

\section{Extended Abstract}

\section{Introduction}

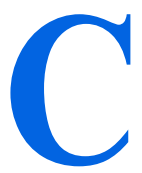

hronic musculoskeletal pain is among the major problems of aging. An effective nursing intervention for pain relief is the use of topical herbal ointments. This study aimed to evaluate the effect of purslane ointment on chronic musculoskeletal pain in older people.

\section{Methods}

This randomized, blinded clinical trial study was conducted in 2017. The study population consisted of all older people with chronic musculoskeletal pain living in Gonabad City, Iran. Of these, 60 were selected using convenience random sampling technique. McGill Pain Questionnaire and the Visual Analogue Scale were used to collect the required data.

\section{* Corresponding Author:}

Kokab Basiri Moghadam, PhD.

Address: Department of Internal and Surgical Nursing and Pediatrics, School of Nursing and Midwifery, Gonabad University of Medical Sciences, Gonabad, Iran.

Tel: +98 (51) 57225027

E-mail: k.basiri@gmu.ac.ir 


\section{Results}

The obtained results suggested that purslane ointment significantly reduced the sensory and emotional dimensions of pain, compared to placebo, in the elderly. The severity of pain in the purslane group significantly decreased after two weeks of intervention, compared to the pre-test scores. The mean pain scores in both sensory and emotional dimensions were not significantly different between men and women (Table 1, 2 \& 3).

\section{Discussion}

A prior randomized clinical trial reported that nipple pain in breastfeeding women significantly reduced on the third and eighth days of lactation when using purslane cream, compared to those receiving lanolin [21]. This finding is consistent with our results. It indicates that purslane is effective in reducing both acute and chronic pain. Farhadpour [28]. evaluated the effect of portulaca oleracea seed hydroalcoholic extract on pain threshold; the palliative and anti-inflammatory effect of purslane were attributed to alpha-tocopherol and its antioxidants which are involved in reducing inflammation and pain by neutralizing the effects of free radicals [28].

The possible mechanism for pain relief is the effect on the peripheral and central nervous system through interaction with GABA receptors [1]. Another possible reason is the inhibition of the release of neurotransmitters [18]. The decreased activity of the musculoskeletal system due to the effect of purslane on the central nervous system and putting peripheral muscles at rest have also been reported in previous studies [18، 28، 29].

The purslane extract and estradiol hormone in appropriate doses increased pain threshold level and increase analgesia in female mice. In addition, a dose-dependent injection of purslane and estradiol extract reduced the pain. It was probably due to the opioid-dependent pathway of endogenous opioids, suggesting the morphine-like effects of the combination of purslane and estradiol extract [26]. The antinociceptive effects of purslane can also be attributed to flavonoid and potassium present in it [28]. The purslane is rich in flavonoids [30].

Table 1. Gender-wise comparison of the sensory and emotional dimensions of pain in the study participants

\begin{tabular}{|c|c|c|c|c|c|c|}
\hline \multirow{2}{*}{ Pain Score } & \multicolumn{2}{|c|}{ Sensory } & \multirow{2}{*}{$\begin{array}{c}\text { Independent } \\
\text { Samples t-test Result }\end{array}$} & \multicolumn{2}{|c|}{ Emotional } & \multirow{2}{*}{$\begin{array}{c}\text { Independent } \\
\text { Samples t-test Result }\end{array}$} \\
\hline & Males & Females & & Males & Females & \\
\hline \multirow{3}{*}{ Mean $\pm S D$} & & & $t=0.781$ & & & $t=0.760$ \\
\hline & $23.17 \pm 2.76$ & $22.60 \pm 2.82$ & $P=0.438$ & $23.37 \pm 2.31$ & $22.92 \pm 2.27$ & $P=0.450$ \\
\hline & & & $d f=58$ & & & $d f=58$ \\
\hline
\end{tabular}

Table 2. Comparing the score of the sensory dimension of pain in both groups

\begin{tabular}{|c|c|c|c|}
\hline \multirow[b]{2}{*}{ Study Phase } & \multicolumn{2}{|c|}{ Mean $\pm S D$} & \multirow{2}{*}{$\begin{array}{l}\text { Independent Samples t-tes } \\
\text { Result }\end{array}$} \\
\hline & Placebo $(n=29)$ & Purslane $(n=31)$ & \\
\hline Baseline & $25.50 \pm 1.24$ & $25.56 \pm 1.34$ & $\begin{array}{c}P=0.848 \\
d f=58 \\
t=0.193\end{array}$ \\
\hline First week & $25.46 \pm 1.23$ & $19.69 \pm 1.33$ & $\begin{array}{c}P<0.001 \\
d f=58 \\
t=-17.38\end{array}$ \\
\hline Second week & $25.41 \pm 1.23$ & $16.30 \pm 1.30$ & $\begin{array}{l}P=0.001 \\
d f=58 \\
t=-27.78\end{array}$ \\
\hline Repeated measures ANOVA & $P=0.81$ & $P=0.001$ & \\
\hline
\end{tabular}


Table 3. Comparing the score of the emotional dimension of pain in both groups

\begin{tabular}{|c|c|c|c|}
\hline \multirow{2}{*}{ Study Phase } & \multicolumn{2}{|c|}{ Mean \pm SD } & \multirow{2}{*}{$\begin{array}{l}\text { Independent Samples t-test } \\
\text { Result }\end{array}$} \\
\hline & Placebo $(n=29)$ & Purslane ( $n=31)$ & \\
\hline \multirow{3}{*}{ Baseline } & & & $P=0.469$ \\
\hline & $25.18 \pm 1.24$ & 24.951 .28 & $\mathrm{df}=58$ \\
\hline & & & $t=-0.728$ \\
\hline \multirow{3}{*}{ First week } & & & $P<0.001$ \\
\hline & $25.13 \pm 1.21$ & $20.66 \pm 1.31$ & $d f=58$ \\
\hline & & & $t=-13.63$ \\
\hline \multirow{3}{*}{ Second week } & & & $P<0.001$ \\
\hline & $25.08 \pm 1.25$ & $18.33 \pm 1.52$ & $d f=58$ \\
\hline & & & $t=-18.61$ \\
\hline Repeated measures ANOVA & $P=0.102$ & $P<0.001$ & \\
\hline
\end{tabular}

Further studies in the field of complementary medicine are recommended with particular attention to medicinal herbs for pain relief and management. Data collection method (self-reporting) was a limitation of this study. The lack of caregiver support for some of the elderly may have also contributed to the severity of pain in these patients.

\section{Conclusion}

The purslane plant reduced the mean pain severity in the purslane group for two consecutive weeks, compared to the placebo group. Therefore, the use of purslane ointment as a safe, cost-effective and feasible intervention is suggested along with therapeutic strategies to reduce chronic musculoskeletal pain among the elderly.

\section{Ethical Considerations}

\section{Compliance with ethical guidelines}

This study is a registered clinical trial (registration code: IRCT20181029041495N1) and obtained its ethical clearance from Gonabad University of Medical Sciences (Code: IR.GMU.REC.1396.100). All ethical guidelines of the Ethics Committee were observed and a written informed consent was obtained from participants

\section{Funding}

This study received financial support from the Student Research Committee of Gonabad University of Medical Sciences.

\section{Authors' contributions}

The authorship contribution rate of first author was 35\%; for the second and third authors, it was $30 \%$ and $35 \%$, respectively.

\section{Conflicts of interest}

The authors declare no conflicts of interests. 


\title{
تأثير يماد خرفه بر دردهاى مزمن اسكلتى عضلاتى سالمندان: يك مطالعه كار آزمايى بالينى تصادفى كورسازي تشده
}

\author{
اميررضا نصيرزاده' (1)، مهدى بصيرى مقدم'، "كوكب بصيرى مقدمم

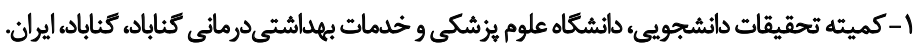

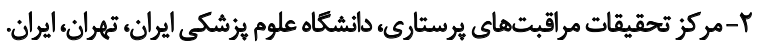

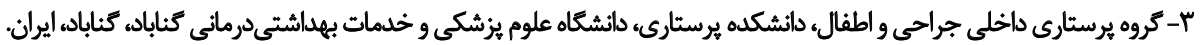

\begin{abstract}
حكSט

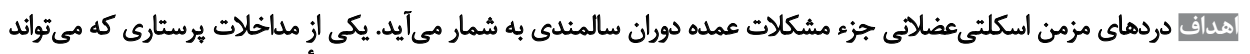

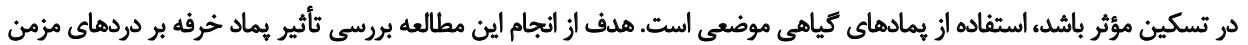

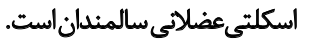

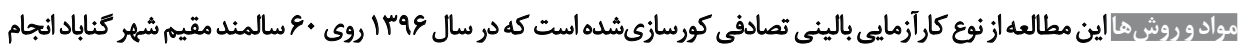

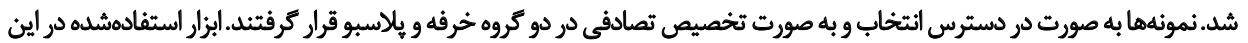

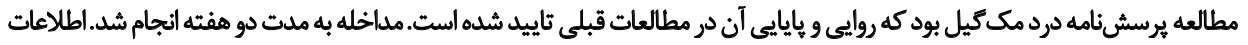

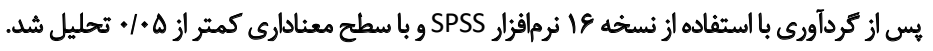

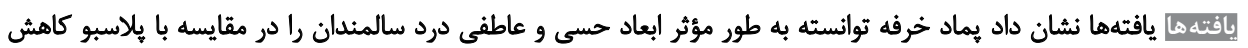

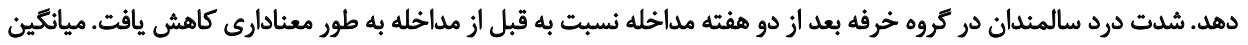

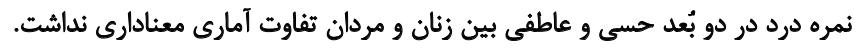

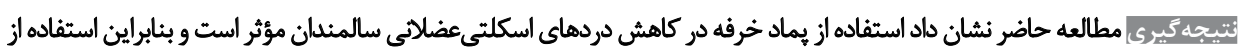

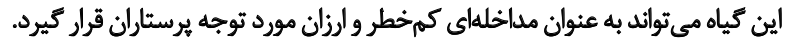

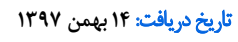

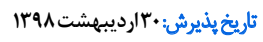

كه عده زيادى از سالمندان از آن ابراز نارضايتى ميكى ديند [ب].

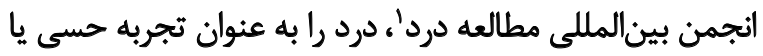

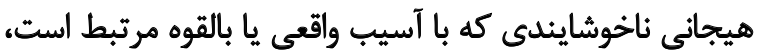

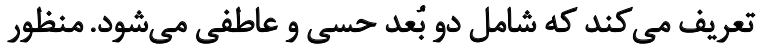

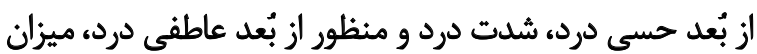

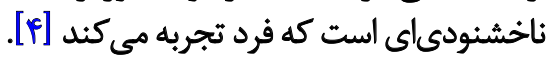

درد از نظر طول مدت نيز به دو دسته درد حاد و مزمن تقسيم

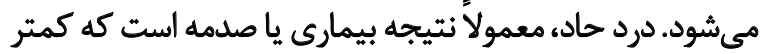

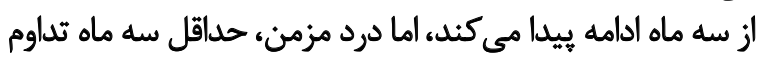

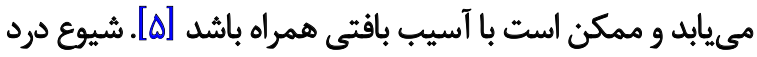

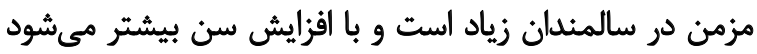

1. Internal Association for the Study of Pain (IASP) doleto

سالمندى يكى از معضلات رو به كسترش در دنياى امروز است

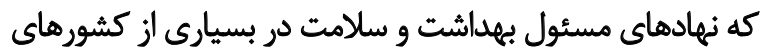

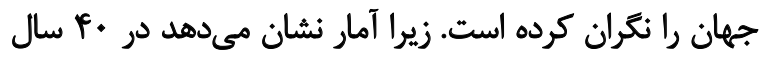

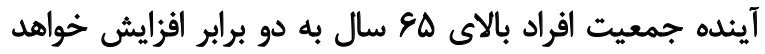

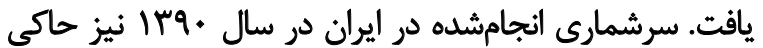

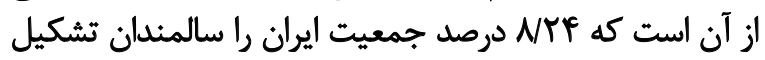

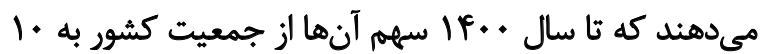
درصد افزايش خواهد يافت [1].

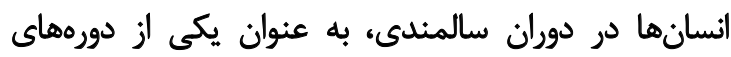

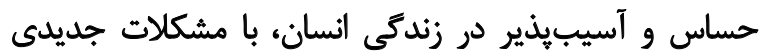

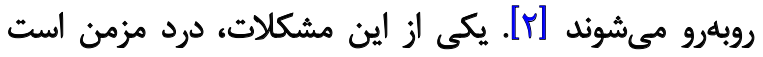


به اينكه خرفه، كياه بومى ايران است و با توجه به فراوانى اين كياه

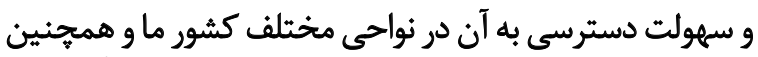

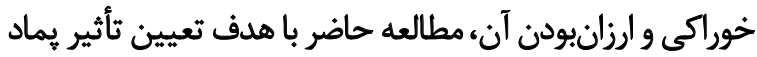
خرفه بر درد مزمن اسكلتى عضلاتى سالمندان انجام شدا.

\section{مواد ورش ها نها}

مطالعه حاضر يك كارآزمايى بالينى تصادفى شده و كورسازىشده

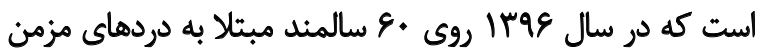

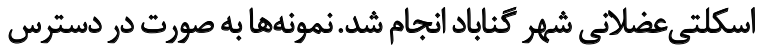

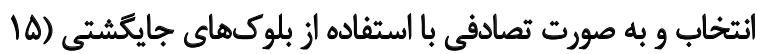

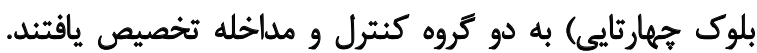

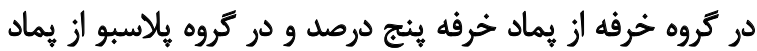

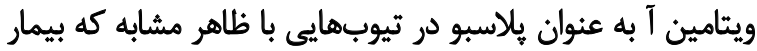

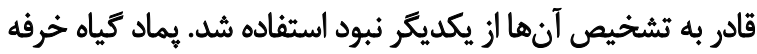

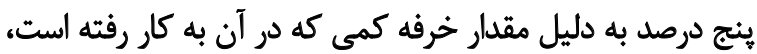

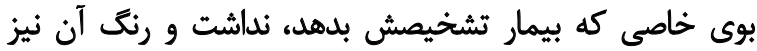

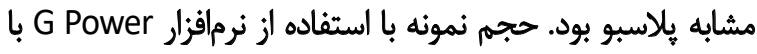

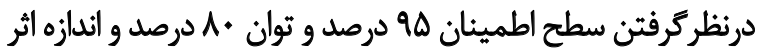

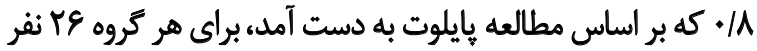

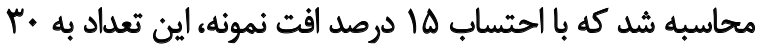

$$
\text { نفر در هر گروه افزايش يافت }
$$

معيارهاى ورود به مطالعه شامل سن •9 سال و بيشتر، ابتلا

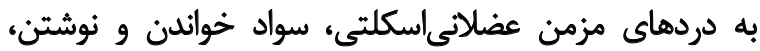

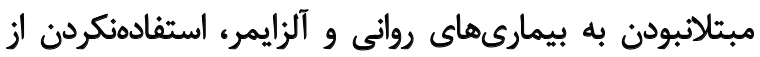

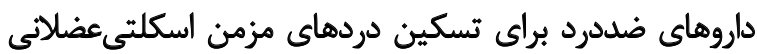

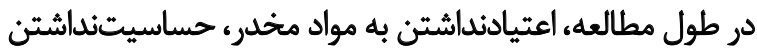

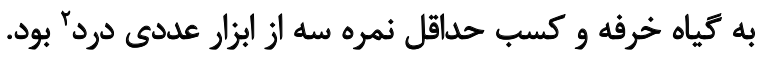

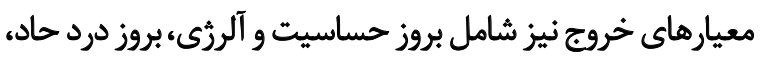
فوت يا همكارىنكردن سالمند بودي.

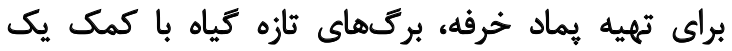

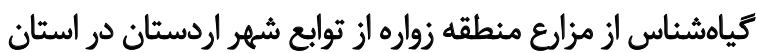

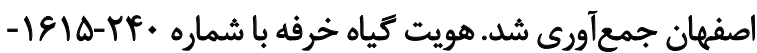

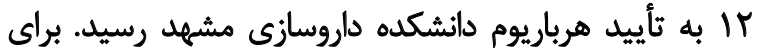

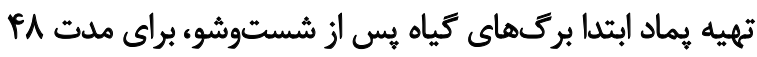

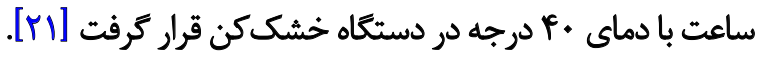

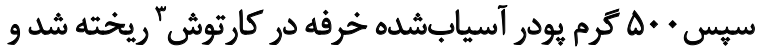

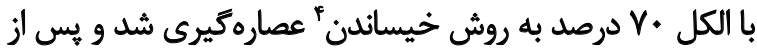

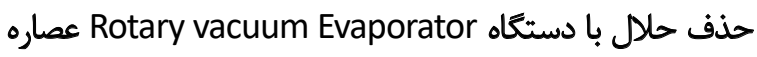

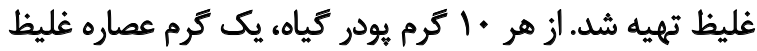

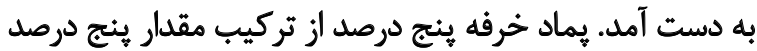

2. Numeric Rating Scale (NRS)

3. Extraction thimbles

4. Maceration

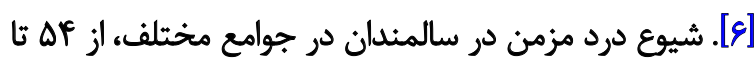

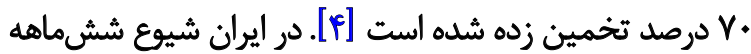

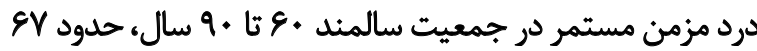

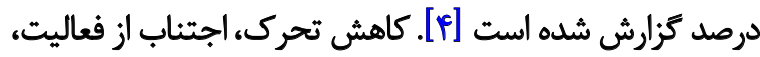

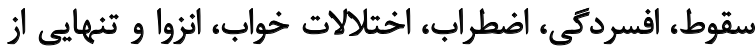

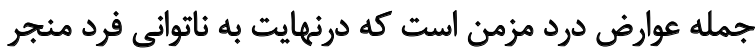

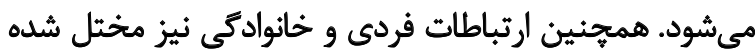

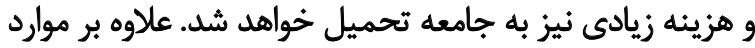

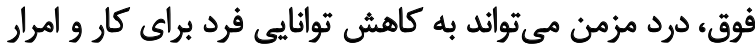

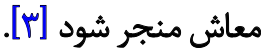

دردهاى اسكلتىعضلانى علت اصلى ناتوانى در سالمندان

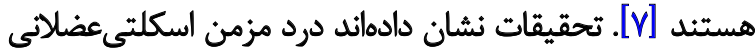

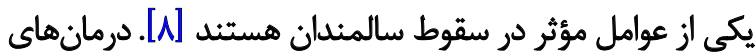

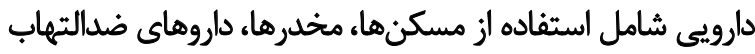

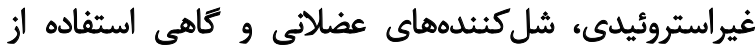

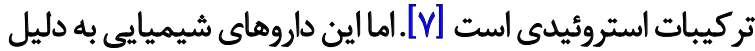

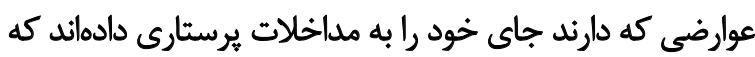

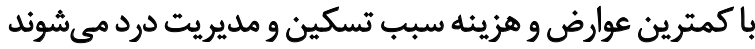

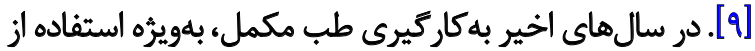

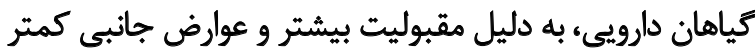

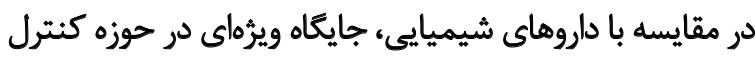
درد ييدا كرده است [.1]].

كياه خرفه يا يريين بانام Purslane و با نام علمى Portulaca ا oleracea

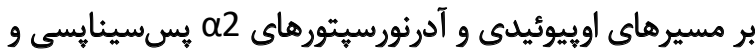

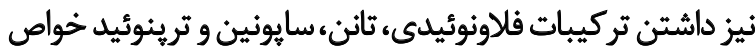

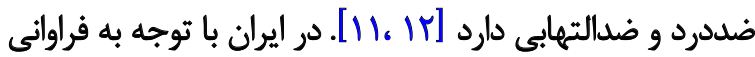

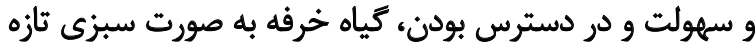
يا خشكشده استفاده مىشود [سا]

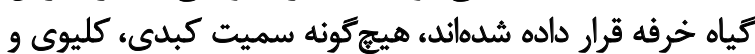

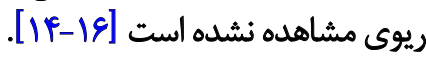
تاكنون اثر سميت سلولى ناشى از عصاره تياه خرفهنيز مشاهده نشده است؛ بنابراين مصرف اين كياه برائ براي بلدن مفيد است وائ واثرات

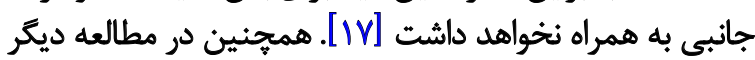

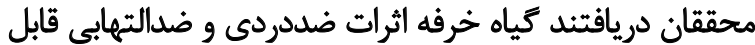

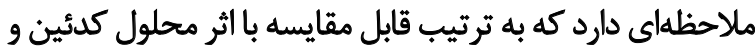

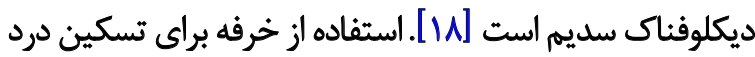

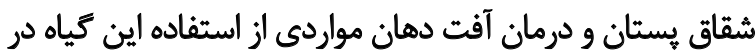
مطالعات انسانى است [. [19.Y]

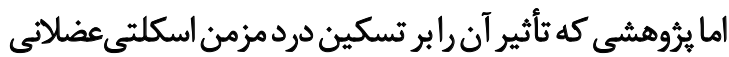

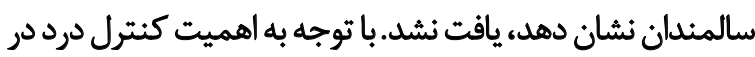

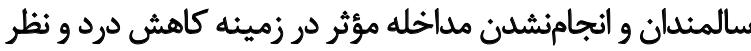




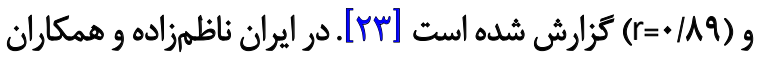

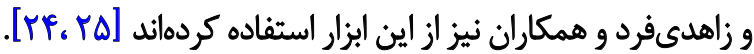

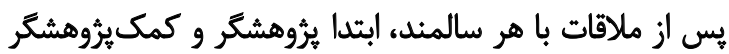

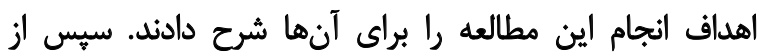

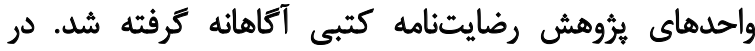

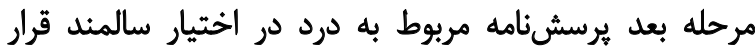

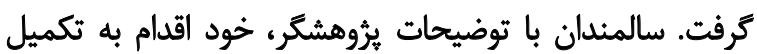

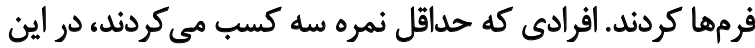

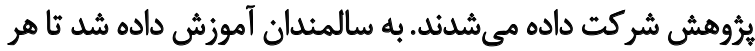

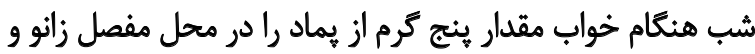

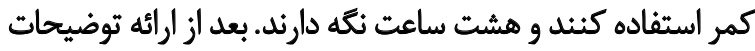

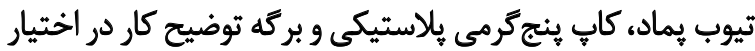

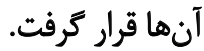

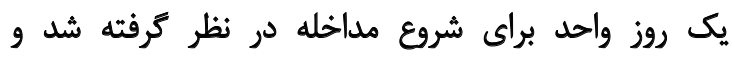

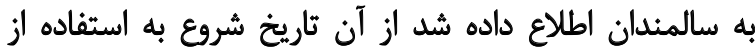

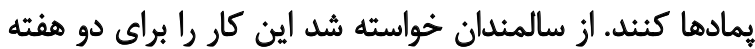

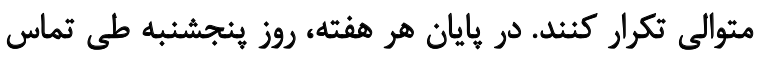

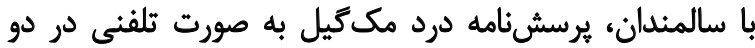

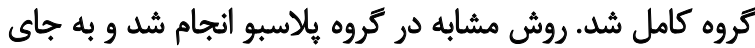

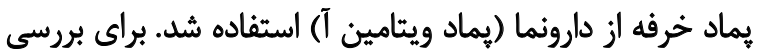

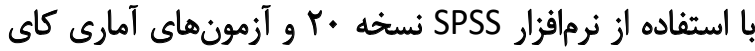

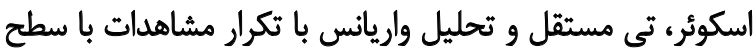

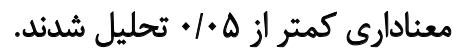

يافتهانها

نتايج مطالعه حاضر نشان داد اعضاى دو كروه از لحاظ متغير هاى

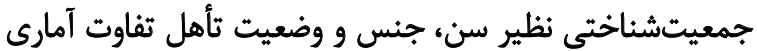

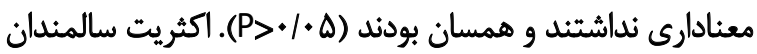

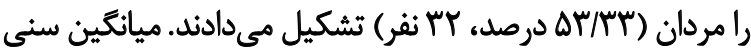

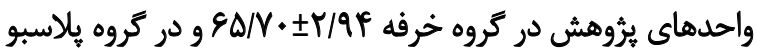
(

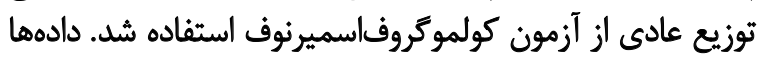

عصاره غليظ كياه خرفه و هو درصد بايه بمادى USP تهيه شد. در اين مطالعه ازي يماد ويتامين آ ساخت كشور ايران و تهيهشده

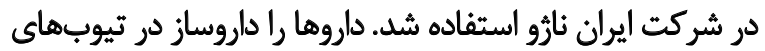

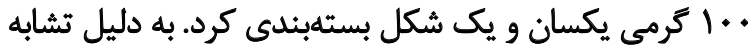

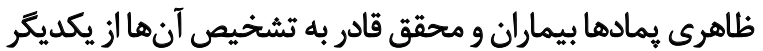

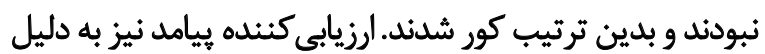

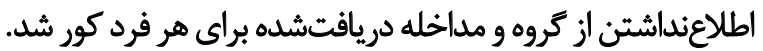

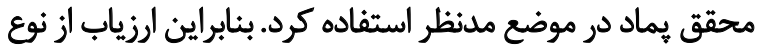

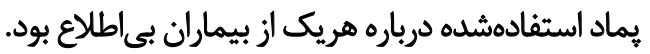

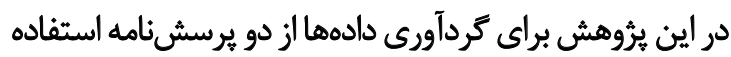

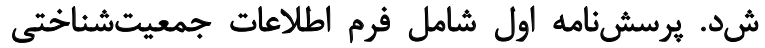

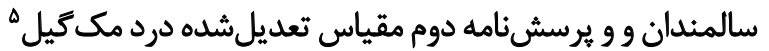

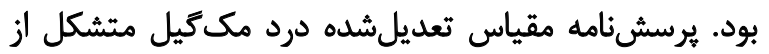

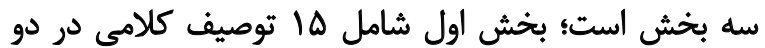

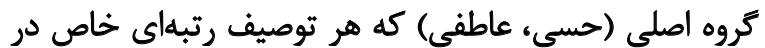

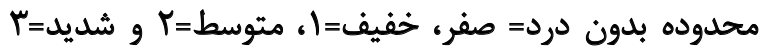

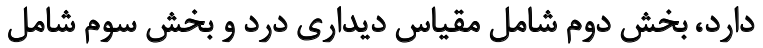

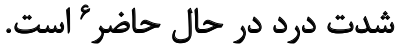

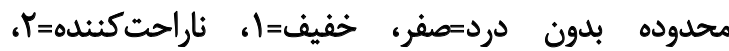

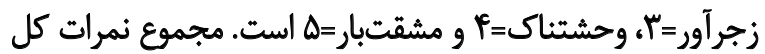

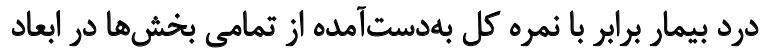

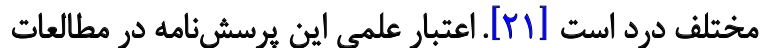

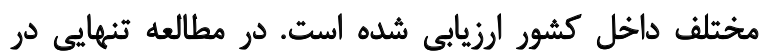

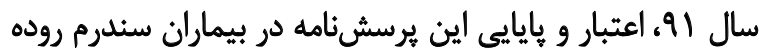

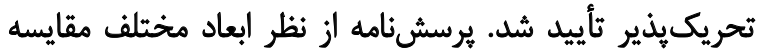

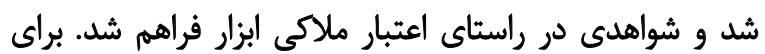

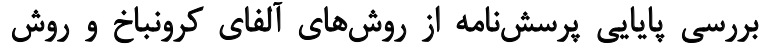

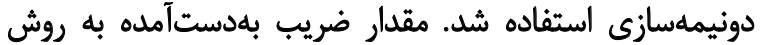

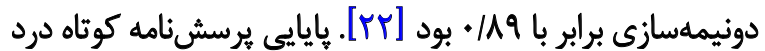

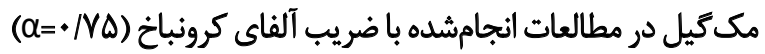

5. McGill

6. Present Pain Intensity (PPI)

جدول ا. مقايسه ميانكين نمره ابعاد حسى و عاطفى درد به تفكيك جنسيت

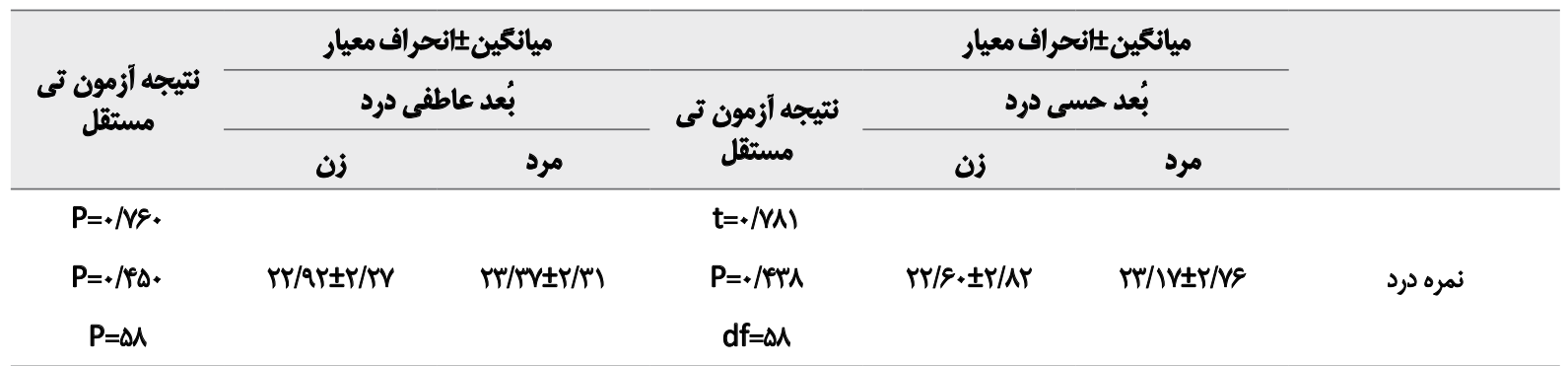

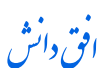




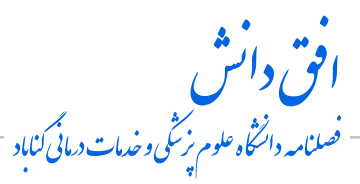

جدول r. مقايسه ميانتين نمره بُعد حسى درد در دو تروه خرفه و يلاسبو در مطالعه

\begin{tabular}{|c|c|c|c|}
\hline \multirow{2}{*}{ آزمون تى مستقل } & \multicolumn{2}{|c|}{ ميانكين } & \multirow{2}{*}{ مراحل } \\
\hline & خرفه (n= (n= & هالاسبو (n=rq) & \\
\hline $\begin{array}{c}P=\cdot / A F A \\
d f=\Delta \Lambda \\
t=\star / / q \mu\end{array}$ & $r \Delta / \Delta C \pm 1 / N r$ & $r \Delta / \Delta \cdot \pm 1 / M r$ & قبل از مداخله \\
\hline $\begin{array}{c}P=/ \cdots 1 \\
d f=\Delta A \\
t=-I V / H A\end{array}$ & $19 / e q \pm 1 / m$ & ra/FEII/M & هفته اول \\
\hline $\begin{array}{c}P=+/ *+1 \\
d f=\Delta A \\
t=-r V N A\end{array}$ & $\mid \varepsilon / \Gamma \cdot \pm 1 / r^{\mu}$ & $r \Delta / f I \pm 1 / \pi r$ & هـته دوم \\
\hline & $P=+1+\infty 1$ & $P=\star|A|$ & آزمون تحليل واريانس با تكرار \\
\hline
\end{tabular}

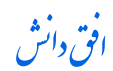

كاهش معنادارى مشاهده نشد (جدول هاي شماره Y و r).

$\stackrel{\leftrightarrow}{4}$

هروهش حاضر با هدف كلى تعيين تأثير بماد خرفه بر بران

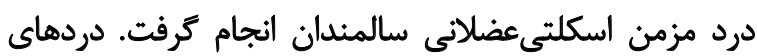

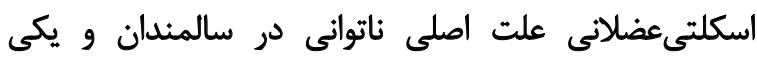

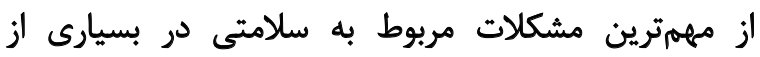

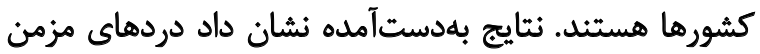

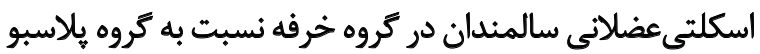

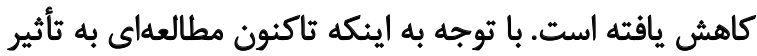

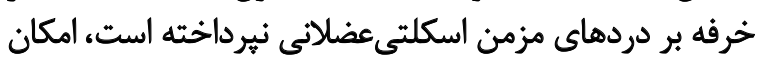

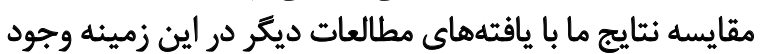

نتيجه آزمون تى مستقل نشان داد بُعد حسى و عاطفى درد بين

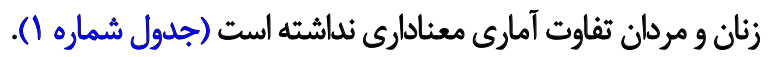

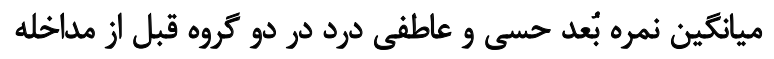

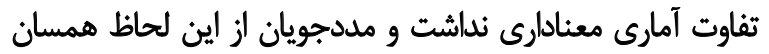

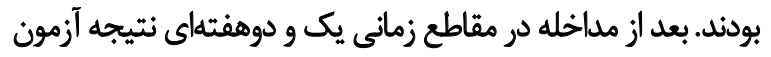

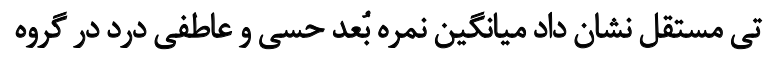

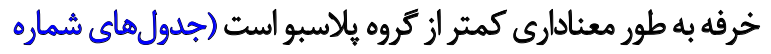

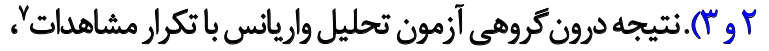

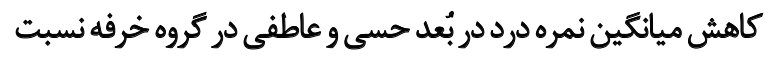

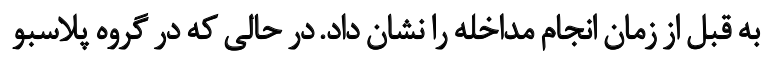

7. Repeated Measures ANOVA

جدول r. مقايسه ميائكين نمره بُعد عاطفى درد در دو كروه خرفه و يلاسبو در طى مطالعه

\begin{tabular}{|c|c|c|c|}
\hline \multirow{2}{*}{ أزمون تى مستثل } & \multicolumn{2}{|c|}{ ميانكين|نانحراف معيار } & \multirow{2}{*}{ مراحل } \\
\hline & خرفه (n=rا & هالاسبو (n=rq) & \\
\hline $\begin{array}{c}P=\cdot / F e q \\
d f=\Delta A \\
t=/ M T A\end{array}$ & $M \Psi / Q \Delta \pm 1 / T \Lambda$ & $r \Delta / M \pm I / T F$ & قبل از مداخله \\
\hline $\begin{array}{c}P<\cdot / \cdots 1 \\
d f=\Delta A \\
t=-\mid r / \& H\end{array}$ & $r \cdot|\& \nexists \pm| / r \mid$ & $r \Delta /|r \pm I / r|$ & هفته اول \\
\hline $\begin{array}{c}P<\cdot / \cdots 1 \\
d f=\Delta A \\
t=-|N \&|\end{array}$ & WNTII/Ar & $r \Delta / \cdot \lambda \pm 1 / r \Delta$ & ل هنثه دوم \\
\hline & $\mathrm{P}<+1++1$ & $P=\cdot / 1 \cdot r$ & أزمون تحليل واريانس با تكرار \\
\hline
\end{tabular}


نتايج بررسى ابعاد حسى و عاطفى درد بين زنان و مردان

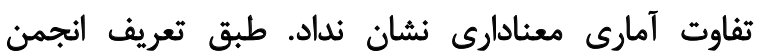

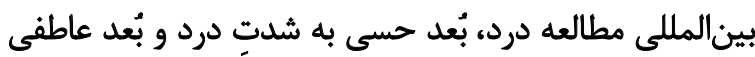

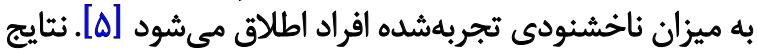

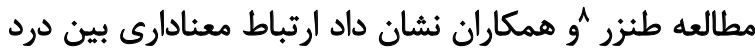

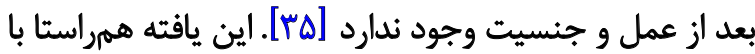

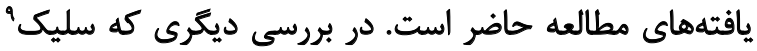

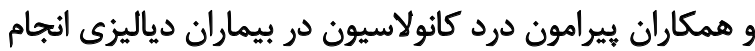

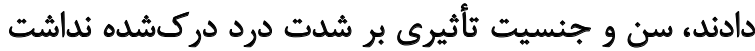

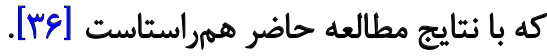

تفاوتنداشتن دو جنس در ميزان درد به دليل تجربه ذهنى

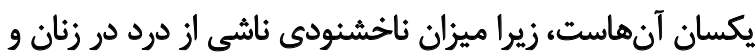

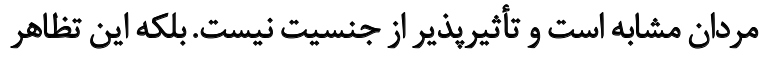

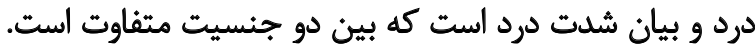

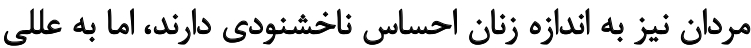

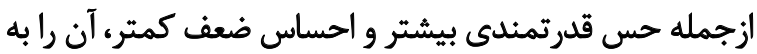

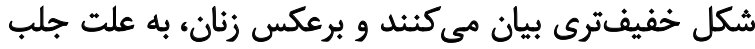
توجه و دريافت حمايت بيشتر درد را شديدتر تزارش مين مي كنيند.

مطالعهاى درباره ذز مرفين استفادهشده براى مردان بعد از

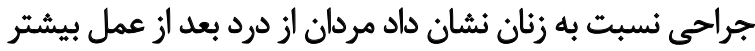

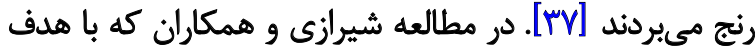

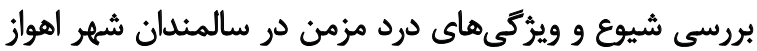

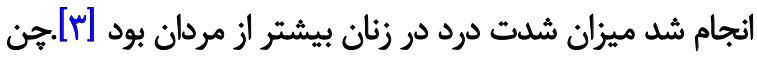

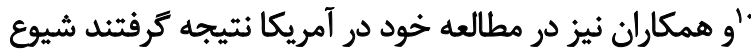

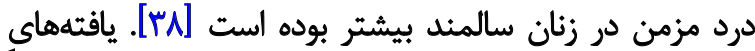

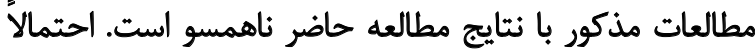

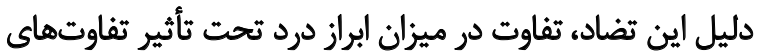

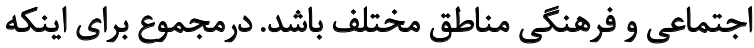

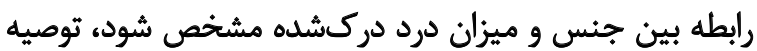

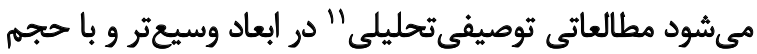

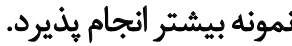

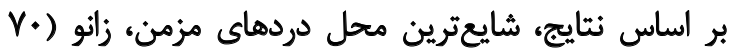

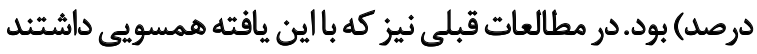

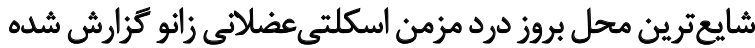

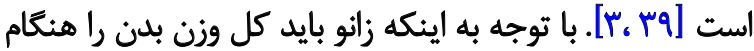

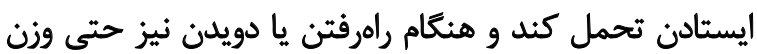

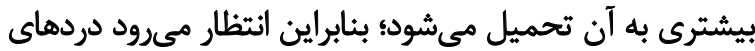

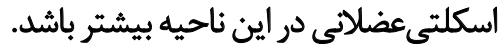

\section{Taenzer}

9. Celik

10. Chen

11. Descriptive-analytical

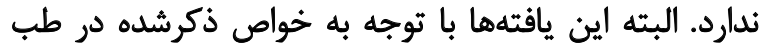

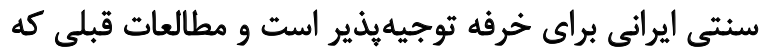

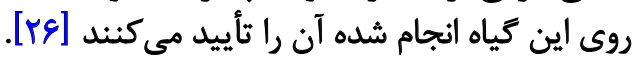
يك مطالعه كارآزمايى بالينى تصادفىشده نشان داد درد

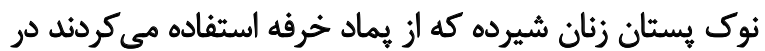

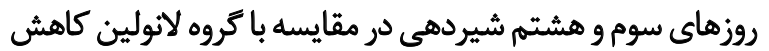

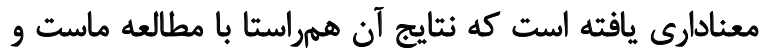

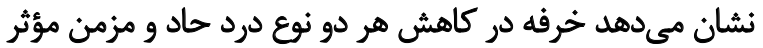

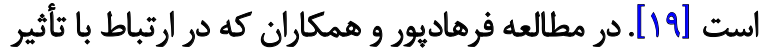

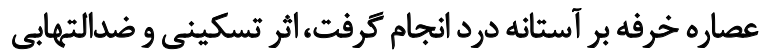

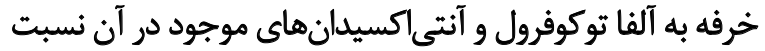

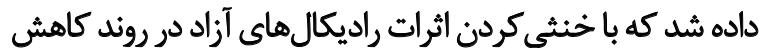

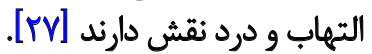
سازوكار احتمالى خرفه براى تسكين درد، تأثيركذارى بر

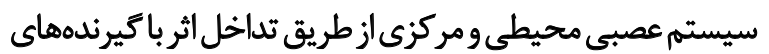

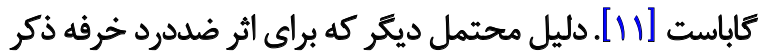

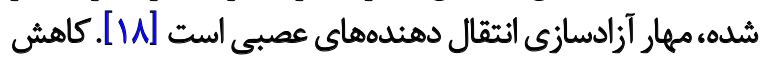

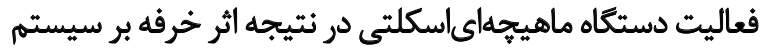

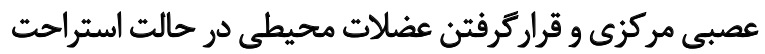

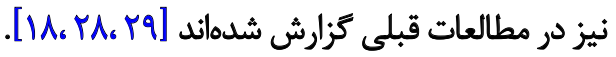
عصاره خرفه و هورمون استراديول در دزهائ مناس مناسب

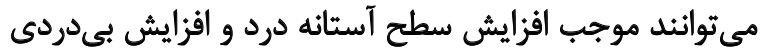

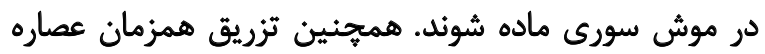

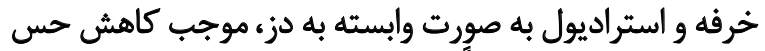

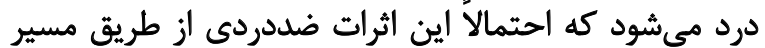

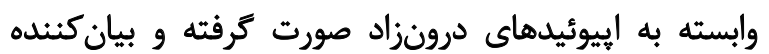

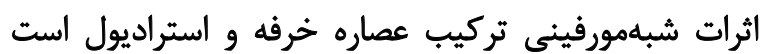

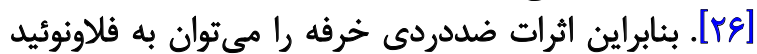

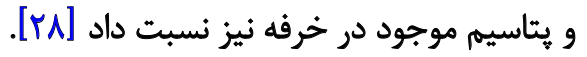

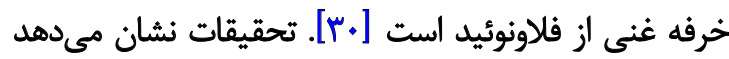

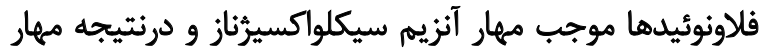

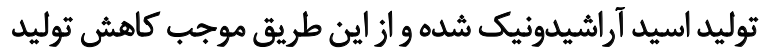

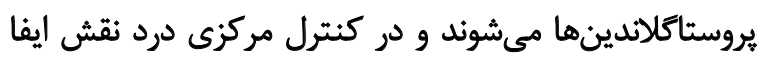

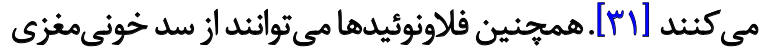

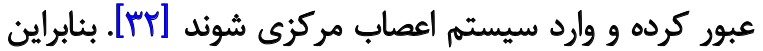

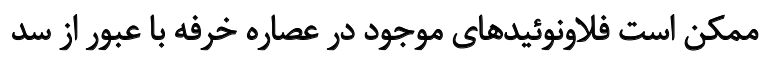

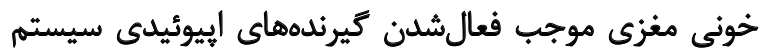

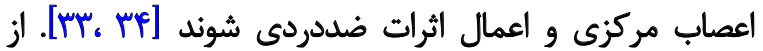

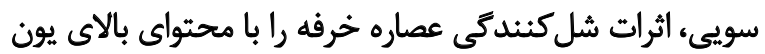

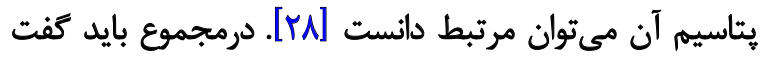

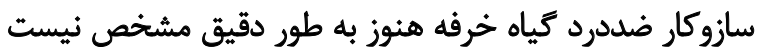

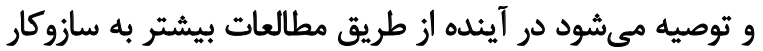

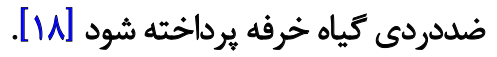




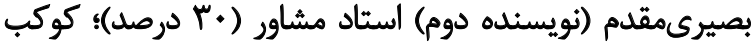
بصيرىمقدم (نويسنده سوم) استاد راهنما (هآ درصد).

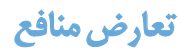
اين طرح فاقد هركونه تعارض منافع است.

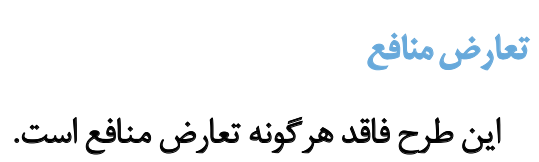

درمجموع مى توان كفت كياه خرفه توانست در دو هفته متوالى

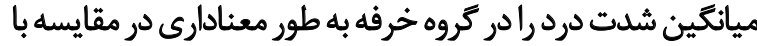

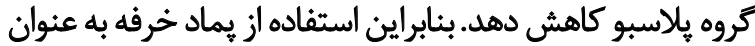

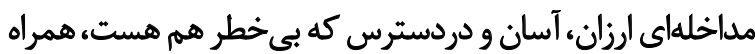

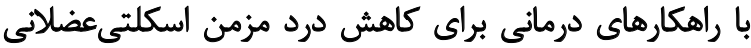

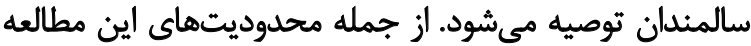

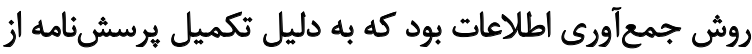

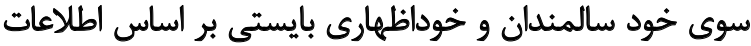

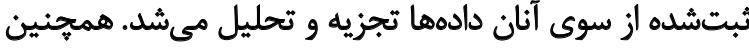

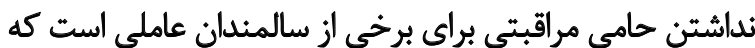

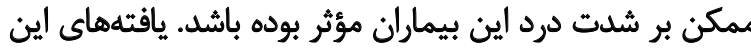

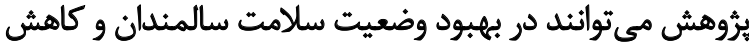

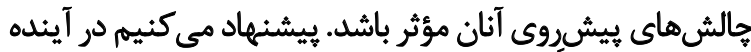

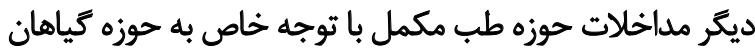

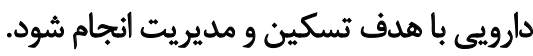

ييشنهاد مىشود مطالعه ديخرى در زمينه تأثير دزهاى بالاتر

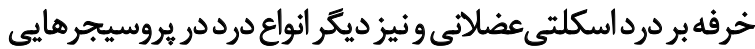

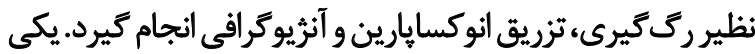

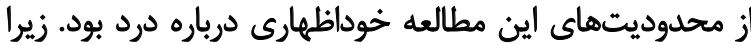

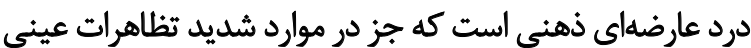

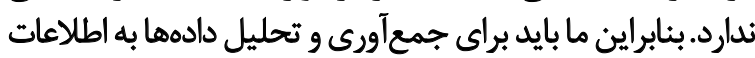
رائهشده از سوى مددجويان تكيه مي بكرديم.

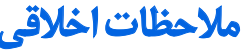

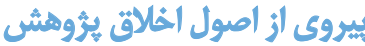

درثوهشكران بعد از تصويب طرح در كميته تحقيقات دانشجويى، كرفتن تأييديه كميته اخلاق دانيك دانشكاه علوم

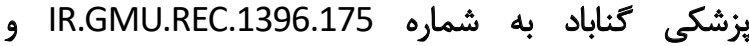
ثبت يروتكل در سايت كارآزمايىهاى بالينى ايران بان به شماره

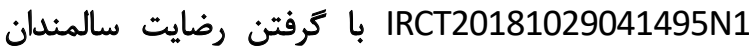
به صورت كتبى و آكاهانه اقدام به نمونهكيرى كردندئ تمام

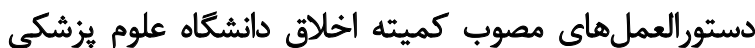
كناباد در جريان انجام مطالعه لحاظ شده است.

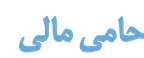

اين بروهش با حمايت مالى كميته تحقيقات دانشجويى دانشكاه علوم يزشكى كناباد انجام شده است.

مشاركت نويسند مكان

اميررضا نصيرزاده (نويسنده اول) يثروهشكر (ه广 درصد)؛ مهلى 
[1] Fotoukian Z, Mohammadi Shahboulaghi F, Fallahi Khoshknab M. [AnaIytical on empowerment interventions in older people with chronic disease: A review literature (Persian)]. Journal of Health Promotion Management. 2013; 2(4):65-76

[2] Salarvand S, Birjandi M, Shamshiri M. [Assessing prevalence of fallings and their relation with chronic conditions for older people living in Khoramabad, Iran (Persian)]. Quarterly of Horizon of Medical Sciences. 2008; 13(4):59-65.

[3] Shirazi M, Manoochehri H, Zagheri Tafreshi M, Zayeri F, Alipour V. [Prevalence of chronic pain and its characteristics among elderly people in Ahvaz City: A cross sectional study (Persian)]. Journal of Gerontological Nursing. 2015; 2(1):63-78

[4] Anbari S, Estaji Z, Rastaqhi S. Assessment effect of rosa damascena juice aromatherapy on elderly chronic musculoskeletal pain in Sabzevar retirement clubs. Iranian Journal of Ageing. 2018; 13(2):250-61. [DOI:10.32598/sija.13.2.250]

[5] Gharaei AS, Azadfallah P, Tavallaei A. [The effectiveness of acceptance and commitment therapy on pain experience in women with chronic pain (Persian)]. Journal of Clinical Psychology. 2012; 4(2):39-50.

[6] Shirazi M, Manoochehri H, Zagheri T, Zayeri F, Alipour V. [Explaining of chronic pain management process in older people: $A$ grounded theory study (Persian)]. Medical-Surgical Nursing Journal. 2016; 4(4):1-10.

[7] Cimmino MA, Ferrone C, Cutolo M. Epidemiology of chronic musculoskeletal pain. Best practice \& Research Clinical Rheumatology. 2011; 25(2):173-83. [DOI:10.1016/j.berh.2010.01.012] [PMID]

[8] Stubbs B, Schofield P, Patchay S, Leveille S. Musculoskeletal pain characteristics associated with lower balance confidence in community-dwelling older adults. Physiotherapy. 2016; 102(2):152-8. [DOI:10.1016/j. physio.2015.03.3721] [PMID]

[9] Salamati A, Mashouf S, Sahbaei F, Mojab F. Effects of inhalation of lavender essential oil on open-heart surgery pain. Iranian Journal of Pharmaceutical Research. 2014; 13(4):1257-61. [PMID] [PMCID]

[10] Afshar M, Fard HS, Shadi M, Ghaderi R. [Repairing effects of Iran flora on wound healing (Persian)]. Journal of Birjand University of Medical Sciences. 2015; 22(1):1-18.

[11] Hajzadeh M, Rakhshandeh H, Esmaeilizadeh M, Ghorbani A. [Analgesic and anti-inflammatory effects of Portolaca oleracea extracts in mice \& rat (Persian)]. Koomesh. 2004; 5(3):113-20.

[12] Rao J, Jayasree T, Mallikarjuna Rao B, Kumar S, Kumar V. Evaluation of the anti-nociceptive and anti-inflammatory activities of the pet: Ether extract of Portulaca oleracea (Linn.). Journal of Clinical and Diagnostic Research. 2012; 6(2):226-30.

[13] Shobeiri S, Sharei S, Heidari A, Kianbakht S. Portulaca Oleracea L. in the treatment of patients with abnormal uterine bleeding: A pilot clinical trial. International Journal Devoted to Pharmacological and Toxicological Evaluation of Natural Product Derivatives. 2009; 23(10):1411-4. [DOI:10.1002/ptr.2790] [PMID]

[14] Eidi A, Mortazavi P, Moghadam JZ, Mardani PM. Hepatoprotective effects of Portulaca oleracea extract against CCl4-induced damage in rats. Pharmaceutical Biology. 2015; 53(7):1042-51. [DOI:10.3109/13880209. 2014.957783] [PMID]

[15] Abd El-Azime AS, Hussein EM, Ashry OM. Synergestic effect of aqueous purslane (Portulaca oleracea L.) extract and fish oil on radiation-induced damage in rats. International Journal of Radiation Biology. 2014; 90(12):1184-90. [DOI:10.3109/09553002.2014.926040] [PMID]
[16] Yue T, Xiaosa W, Ruirui Q, Wencai S, Hailiang X, Min L. The effects of Portulaca oleracea on hypoxia-induced pulmonary edema in mice. High Altitude Medicine \& Biology. 2015; 16(1):43-51. [DOI:10.1089/ ham.2013.1081] [PMID] [PMCID]

[17] Yen G, Chen H, Peng H. Evaluation of the cytotoxicity, mutagenicity and antimutagenicity of emerging edible plants. Food and Chemical Toxicology. 2001; 39(11):1045-53. [DOI:10.1016/S0278-6915(01)00053-9]

[18] Boroushaki MT, Boskabady MH, Malek F. Antitussive effect of Portulaca oleracea L. In guinea pigs. Iranian Journal of Pharmaceutical Research. 2010; 3(3):187-90. [DOI:10.22037/IJPR.2010.599]

[19] Niazi A, Yousefzadeh S, Rakhshandeh H, Esmaeili H. [Comparison of purslane cream and lanolin on nipple pain among breastfeeding women: A randomized clinical trial (Persian)]. The Iranian Journal of Obstetrics, Gynecology and Infertility. 2018; 20(12):77-85. [DOI:10.22038/ IJOGI.2017.10433]

[20] Najafi S, Mohammadzadeh M, Monsef Esfahani H, Meighani G, Rezaei $\mathrm{N}$. [The effect of Purslane in the treatment of recurrent aphthous stomatitis (Persian)]. Tehran University Medical Journal. 2013; 71(2):102-8.

[21] Niazi A, Yousefzadeh S, Rakhshandeh H, Esmaeily H. [The effect of nipple soreness treatment with Purslane cream and lanolin on frequency and duration of breastfeeding in nursing mothers: A randomized clinical trial (Persian)]. Journal of Midwifery and Reproductive Health. 2019; $7(1): 1527-35$.

[22] Jaafarnejad F, Hosseini SF, Mazloom SR, Hami M. [Comparison of the Effect of Fish Oil and Vitamin E on the Severity of Cyclic Mastalgia (Persian)]. The Iranian Journal of Obstetrics, Gynecology and Infertility. 2013; 15(39):9-15.

[23] Tanhaee Z, Fathi-Ashtiani A, Amini M, Vahedi H, Shaghaghi F. [Validation of a revised version of the Short-Form Mc-Gill Pain Questionnaire (SF-MPQ-2) for IBS patients (Persian)]. Govaresh. 2012; 17(2):91-7.

[24] Quinn F, Hughes CM, Baxter G. Reflexology in the management of low back pain: A pilot randomised controlled trial. Complementary Therapies in Medicine. 2008; 16(1):3-8. [DOI:10.1016/j.ctim.2007.07.004] [PMID]

[25] Zahedifard T, Firozi M. [Assessment of the articles related to primary dysmenorrhea in dimension of menstrual pain assessment tools (Persian)]. The Iranian Journal of Obstetrics, Gynecology and Infertility. 2016; 19(4):17-27

[26] Keshavarzi K, Moradi M, Khoshnam SE, Alvani A, Safaei Pour Zamani $M$, Fatahi $M$, et al. [Evaluation the effect of hydro-alcoholic extract of portulaca oleracea and estradiol on pain threshold in female mice (Persian)]. Journal of Medicinal Plants. 2017; 2(62):29-37.

[27] Farhadpour F, Alvany A, Khakpour B, Ahmadi R, Mahdavi E. The effects of Portulaca Oleracea seed hydroalcoholic extract on pain threshold. Journal of Biomedical Science. 2014; 10:80-1.

[28] Radhakrishnan R, Zakaria MN, Islam MW, Chen HB, Kamil M, Chan $\mathrm{K}$, et al. Neuropharmacological actions of Portulaca Oleraceae L V. sativa (Hawk). Journal of Ethnopharmacology. 2001; 76(2):171-6 [DOI:10.1016/S0378-8741(01)00230-6]

[29] Rhudy JL, Bartley EJ, Palit S, Kerr KL, Kuhn BL, Martin SL, et al. Do sex hormones influence emotional modulation of pain and nociception in healthy women? Biological Psychology. 2013; 94(3):534-44. [DOI:10.1016/j.biopsycho.2013.10.003] [PMID]

[30] Schölly T, Kapetanidis I. Flavonol and naphthoquinone glycosides of Drosera rotundifolia. Planta Medica. 1989; 55(07):611-2. [DOI:10.1055/s-2006-962162] 
[31] Nijveldt RJ, Van Nood E, Van Hoorn DE, Boelens PG, Van Norren K, Van Leeuwen PA. Flavonoids: A review of probable mechanisms of action and potential applications. The American Journal of Clinical Nutrition. 2001; 74(4):418-25. [DOI:10.1093/ajcn/74.4.418] [PMID]

[32] Loscalzo LM, Wasowski C, Paladini AC, Marder M. Opioid receptors are involved in the sedative and antinociceptive effects of hesperidin as well as in its potentiation with benzodiazepines. European Journal of Pharmacology. 2008; 580(3):306-13. [DOI:10.1016/j.ejphar.2007.11.011] [PMID]

[33] Grasshoff C, Drexler B, Rudolph U, Antkowiak B. Anaesthetic drugs: Linking molecular actions to clinical effects. Current Pharmaceutical Design. 2006; 12(28):3665-79. [DOI:10.2174/138161206778522038] [PMID]

[34] Nishiyama T. Analgesic effects of systemic midazolam: Comparison with intrathecal administration. Canadian Journal of Anesthesia. 2006; 53(10):1004-9. [DOI:10.1007/BF03022529] [PMID]

[35] Taenzer AH, Clark C, Curry CS. Gender affects report of pain and function after arthroscopic anterior cruciate ligament reconstruction. Anesthesiology. 2000; 93(3):670-5. [DOI:10.1097/00000542-20000900000015] [PMID]

[36] Çelik G, Özbek O, Yılmaz M, Duman I, Özbek S, Apiliogullari S. Vapocoolant spray vs lidocaine/ prilocaine cream for reducing the pain of venipuncture in hemodialysis patients: A randomized, placebo-controlled, crossover study. International Journal of Medical Sciences. 2011; 8(7):623-7. [DOI:10.7150/ijms.8.623] [PMID] [PMCID]

[37] Chia YY, Chow LH, Hung CC, Liu K, Ger LP, Wang PN. Gender and pain upon movement are associated with the requirements for postoperative patient-controllediv analgesia: A prospective survey of 2,298 Chinese patients. Canadian Journal of Anesthesia. 2002; 49(3):249. [DOI:10.1007/BF03020523] [PMID]

[38] Chen Q, Hayman LL, Shmerling RH, Bean JF, Leveille SG. Characteristics of chronic pain associated with sleep difficulty in older adults: the Maintenance of Balance, Independent Living, Intellect, and Zest in the Elderly (MOBILIZE) Boston study. Journal of the American Geriatrics Society. 2011; 59(8):1385-92. [DOI:10.1111/j.1532-5415.2011.03544.x] [PMID] [PMCID]

[39] Alipour M. [The relationship between chronic musculoskeletal pain and vitamin d deficiency in the elderly population of Amirkola, Iran (Persian)]. Journal of Babol University of Medical Sciences. 2015; 17(10):7-14. 
This Page Intentionally Left Blank 